\title{
Smart Boiler System at Power Generation Plant using Power Line Carrier Communication
}

\author{
Nagesh Jadhav \\ Student, Dept. of ENTC \\ Dr.D.Y.Patil school of engineering, \\ Lohegaon \\ Pune, Maharashtra, India
}

\author{
Sonali Khande \\ Student, Dept. of ENTC \\ Dr.D.Y.Patil school of engineering, \\ Lohegaon \\ Pune, Maharashtra, India
}

\author{
Yogesh Shinde \\ Assistant Professor, Dept. of ENTC \\ Dr.D.Y.Patil school of engineering, \\ Lohegaon \\ Pune, Maharashtra, India
}

\begin{abstract}
Electrical power network that covers almost all over the places in the world. Electrical power network can be utilized as a communication medium; it is possible due to its widely spread network and connectivity. The trending technology of communicating over the power line network is known to everyone since 19th century. The main aim of this project to propose low cost system hardware for controlling and monitoring boiler system at the power generation plant using power line carrier communication (PLCC) technology. The proposed system allows them to full control on the power generation plant with less effort. PLCC technology that enables sending and receiving data over AC power line. New modulation technologies are widely used ins power line technology. Due to the ease of installation, inexpensive, high speed communication medium, availability of AC cables, low cost, high reliability and security the PLCC is widely preferred over the wireless communication or other short distance communication technologies. PLCC technology is better than GSM technology due to ease of installation, availability of network, low cost of installation, high reliability.
\end{abstract}

\section{Keywords}

Smart Boiler System

\section{INTRODUCTION}

While preparing a communication system, we have to provide extra cables and special encoding techniques. In order to reduce this type of work, we have to send the communication signals along with the AC power line. This can be achieved by Power Line Carrier Communication (PLCC) technology. This technology is used in the automated meter reading, substation level, home automation, etc [8]. The research is ongoing to implement PLCC in various applications such as economical and industrial application. We are proposed a system to implement the PLCC for boiler system at power generation plant. PLCC can be used in both AC and DC power line cables. Recently PLCC is evolved as a cognitive radio networks and this network used to avoid the spectrum deficiency in communication signals [1]. PLCC technology provides successful transmission in both narrowband and broadband communication [3].

PLCC technology is more suitable for substation and distribution electrical power networks than the other technologies like WIFI, WIMAX, and ZIGBEE etc [4]. PLCC technology is used instead of fiber optic and wireless networks in smart grid systems [11]. In this paper we are simulated the model to control and monitor boiler system parameters at power generation plant.

\section{POWER LINE CARRIER COMMUNICATION}

PLCC means simply the information signal is transferred over the already existing electrical power line wiring infrastructure. It is also called as power line communication, main communication, power line digital subscriber line and power line networking. PLCC technology has been established from many years but it is still not used in the home and small industry application. The information signals are transmitted by superimposing with the $50 \mathrm{~Hz}$ electrical signal frequency. In PLCC technology various modulation techniques used are as Frequency Shift Keying (FSK), Amplitude Shift Keying (ASK), Phase Shift Keying (PSK), Orthogonal Frequency Division Multiplexing (OFDM), Coded Orthogonal Frequency Division Multiplexing (COFDM), Spread Spectrum (SS) and Gaussian Minimum Shift Keying (GMSK) [10]. PLCC systems consist of a high frequency signal injection over the electrical power lines [6].

\section{SYSTEM DESCRIPTION}

This system is proposed to use in the small industries specifically for the boiler system. To control and monitor the entire boiler system through power line network with the help of power line carrier communication. This proposed system saves complex wiring installation, human efforts and human resource at the power generation plant. The control room acts as host station for transmitting the controlling signal and receiving the signals from boiler system to monitor the parameters and PLCC modem is acts as transceiver serially using recommended standard (RS232). PLCC modem uses the regular power outlet to transmit and receive the controlling signal. The parameters required for boiler system collected by the different sensors and then it send to control room through PLCC.

\section{SYSTEM DIAGRAM}

A. Control room section

Figure (a) proposed the block diagram of control room section. The control room section operates as a transmitter and receiver. If the keypad generates the controlling signal then this system acts as a transmitter and if it receives the boiler parameters then it acts as receiver. In this proposed system microcontroller is used control send and receive the signals for successful transmission. 


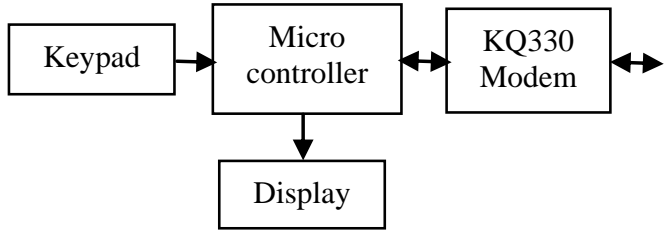

Figure (a): Block diagram of control room system section.

The command signal is send in the form of characters or numbers to control the boiler system section i.e. ON or OFF water heater, water motor, etc. The microcontroller converts the command signal into serial digital signals. This data is merged with the help of zero crossing detector in PLCC modem and transmit over the AC power line. This section receives the information signal from boiler system section to continuously monitor the parameters of boiler system.

B. Boiler system section.

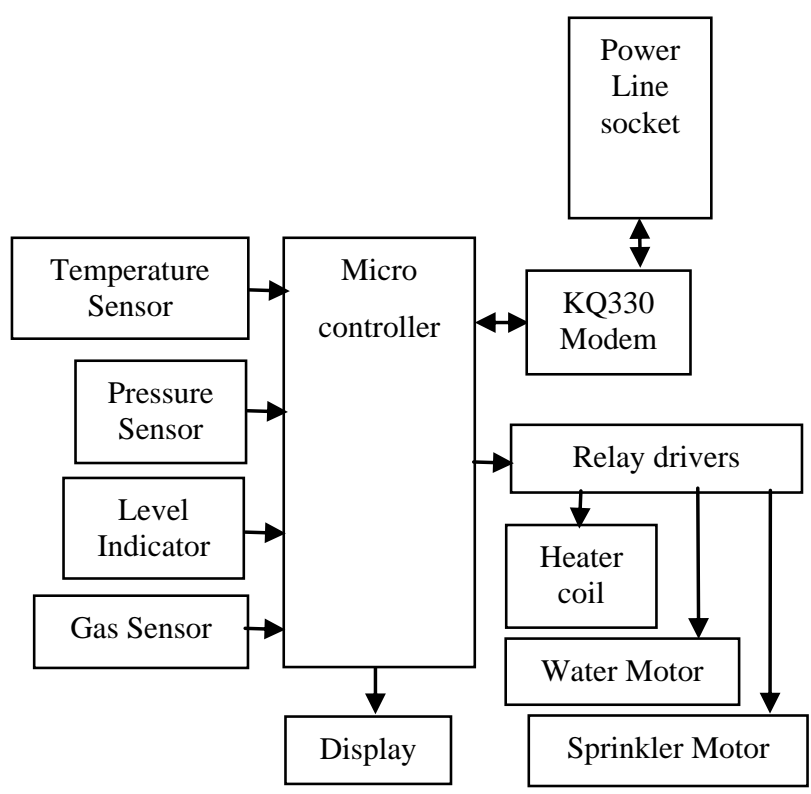

Figure (a): Block diagram of boiler system section.

The boiler system section also operates as a both transmitter and receiver. The transmitted command signal from control room received by the PLCC modem at the boiler system section. PLCC modem converted it into serial digital signal. This serial digital data signals are sending to the microcontroller. Then microcontroller gives command signal to the relay driver for which equipment have to be control. And then it sent to display section for monitoring purpose. The sensors connected with boiler system collects data and send to the microcontroller to convert it into a digital data signal. Then this digital data send over the power line with the help of PLCC modem.

\section{B. PLCC Modem}

PLCC will modulate and demodulate the data on the AC signal and it is cost effective modem. It has $9600 \mathrm{bps}$ interface baud rate. This module has two types of operating mode. It uses a transparent mode when control module MODE pin set to high level, or a custom operating mode when set to low level. It connected easily with the microcontroller and AC power line. No need to initialize the module programming, normal serial communication type similar manner programming. PLCC modem sends data in the format of a start bit, 8 data bits, 1 stop bit format. KQ330 modem is a complete carrier module, and transmitting data independent of the zero crossing detector. After carrier demodulation digital filtering done to improve its anti-interference ability of the data carrier.

\section{HARDWARE}

A. Control room section

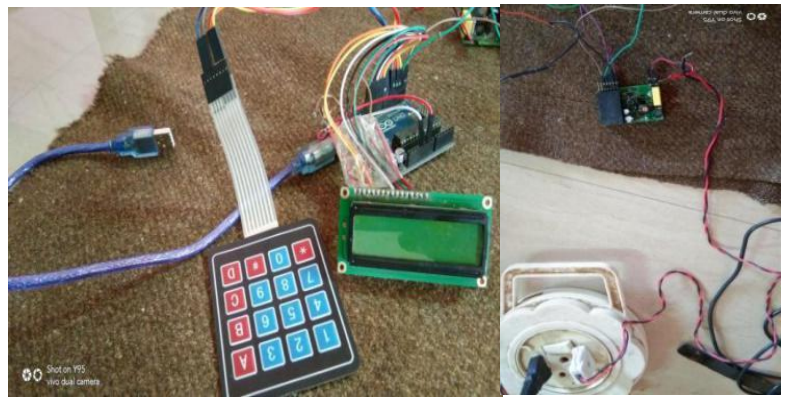

B. Boiler system section.

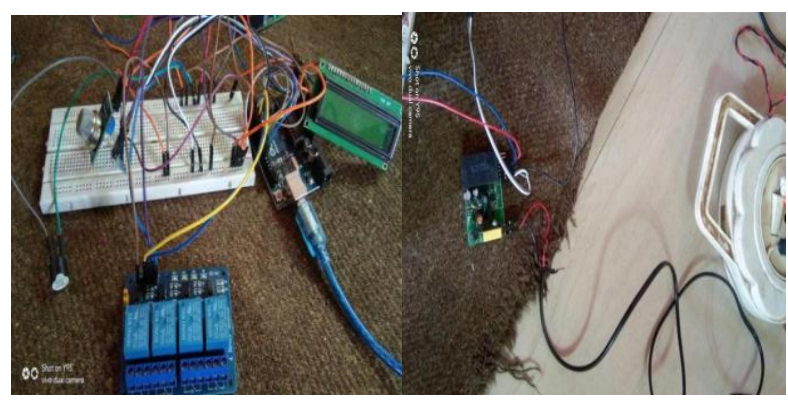

6. RESULT

A. Monitoring all parameters of boiler system

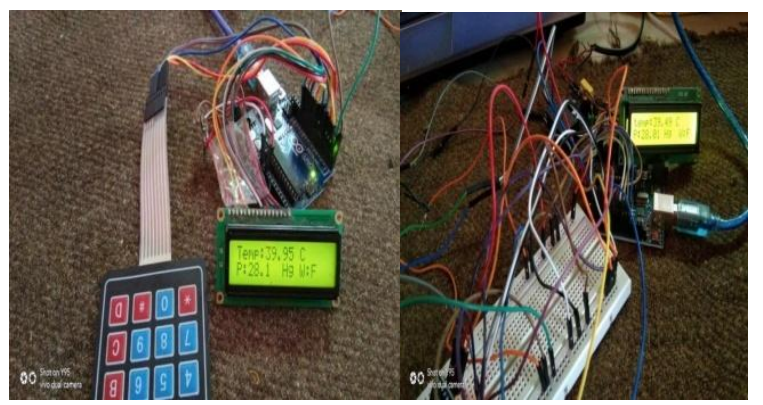

We are mainly focusing on tree parameters of the boiler system i.e. temperature, pressure, and water level. As shown in these parameters are continuously shown on the display at the both section of entire proposed system.

B. Setting temperature for boiler system

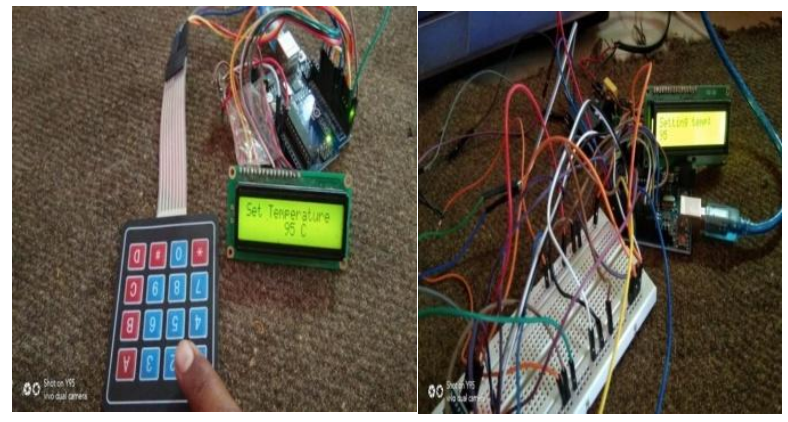

With the help of keypad at the control room section we can set the temperature according to the requirement. According to 
temperature value the entire system will works till it does changed from control room.

\section{CODING}

In this proposed system two different codes for control room section and boiler system section. At boiler system section it continuously collect values from sensor and send towards the control room section but if there is an signal receive from gas sensor then shuts down entire the system and only display unit shows the safety notifications. When command signal received from control room section, boiler system parameters are set to particular values. The monitoring of parameters is possible at both the sections.

\section{CONCLUSION}

In this proposed system of power line carrier communication we concluded that KQ330 module has a better transmission distance over a power line. Wireless communication is costly and short distance and also it is not strong in the winter season as compare to PLCC technology. Power line carrier communication is an advanced way of communication technique. As compared to wireless technology, it transmits signal over the longer distance and also it can receive any data at any power terminal connected in same electrical power network.

\section{REFERENCES}

[1] Lavanya.V, Poovizhi. I, Vimala. P "Power Line Carrier Communication for Home and Small Industries based on the Low Cost "SSRG International Journal of Electrical and Electronics Engineering (SSRG - IJEEE), Volume 4 Issue 3, March 2017

[2] Abdul Mannan, D. K. Saxena, Mahroosh Banday "A Study on Power Line Communication" International Journal of Scientific and Research Publications, Volume 4, Issue 7, July 2014

[3] Dipalee M. Kate, Sanket Sunil Mange "Power Line Communication for Real Time Devices Control" International Journal of Advanced Research in Electrical, Electronics and Instrumentation Engineering Vol. 6, Issue 6, June 2017
[4] Rashmi Sharma "PLC based Data Transmission System" International Journal of Innovative Research in Science, Engineering and Technology Vol. 7, Issue 6, June 2018

[5] Xiaoqiang Xua, Ao Zhana, Xiaohan Lia "Design and implementation of street light control system based on power line carrier communication" The 5th International Workshop on Wireless Technology Innovations in Smart Grid (WTISG) August 19-21, 2019

[6] Kyung Mog Lee "Construction of a Home Network System Using a Power Line Communication Method and a Voice Recognition Command Controller" Proceedings of the World Congress on Engineering and Computer Science 2013 Volume II WCECS 2013, 23-25, October 2013

[7] Joshin P.C., Melvin Joseph, Sam James, Vishnu Sasidharan "Automation using Power Line Communication with Web Based Access" International Journal of Advanced Research in Electrical, Electronics and Instrumentation Engineering An ISO 3297: 2007 Certified Organization Vol. 4, Special Issue 1, March 2015

[8] J. Manikandan, V. Ramya "Design and Implementation of Smart Home Control System Based on Power Line Communication" International Conference on Engineering Innovations and Solutions, March 2016

[9] Sai Vishal, S.T. Aarthy, Tungala Sai Puneeth, Neelisetty Jagadeesh "Design and Implementation of Smart Home control systems using Power Line Communication (PLC)" International Journal of Pure and Applied Mathematics Volume 115 No. 7 2017, 577-583, June 2017

[10] Nidzamudeen Ishak, Norashida Md Din, Md Zaini Jamuldin and Yasmin Hanum Mohd "Power line carrier technology and home automation", IEEE transaction, 2002.

[11] Stefano Galli, Anna Scaglione, Zhifang Wang "Power Line Communications and the Smart Grid" IEEE J. Sel. Areas Communication, vol. 24, no. 7, Jul. 2014. 